\title{
Correction to: Functional variation in allelic methylomes underscores a strong genetic contribution and reveals novel epigenetic alterations in the human epigenome
}

Warren A. Cheung ${ }^{1,2+}$, Xiaojian Shao ${ }^{1,2+}$, Andréanne Morin ${ }^{1,2+}$, Valérie Siroux ${ }^{3}$, Tony Kwan ${ }^{1,2}$, Bing Ge ${ }^{1,2}$, Dylan Aïssi ${ }^{3}$, Lu Chen ${ }^{4,5}$, Louella Vasquez ${ }^{4}$, Fiona Allum ${ }^{1,2}$, Frédéric Guénard ${ }^{6}$, Emmanuelle Bouzigon ${ }^{7}$, Marie-Michelle Simon², Elodie Boulier ${ }^{2}$, Adriana Redensek ${ }^{2}$, Stephen Watt ${ }^{4}$, Avik Datta $^{8}$, Laura Clarke ${ }^{8}$, Paul Flicek ${ }^{8}$, Daniel Mead ${ }^{4}$, Dirk S. Paul ${ }^{9,10}$, Stephan Beck ${ }^{9}$, Guillaume Bourque ${ }^{1,2}$, Mark Lathrop $^{1,2}$, André Tchernof ${ }^{11}$, Marie-Claude Vohl ${ }^{6}$, Florence Demenais ${ }^{7}$, Isabelle Pin ${ }^{3,12}$, Kate Downes ${ }^{5,13}$, Hendrick G. Stunnenberg ${ }^{14}$, Nicole Soranzo ${ }^{4,5,15,16}$, Tomi Pastinen ${ }^{1,2^{*}}$ and Elin Grundberg ${ }^{1,2^{*}}$

\section{Correction to: Genome Biol}

https://doi.org/10.1186/s13059-017-1173-7

Following publication of the original article [1], the authors reported an error in Additional file 1. The updated Additional file 1 is given below.

\section{Additional file}

Additional file 1 Description of MCC-Seq capture panel. This file contains: (1) summary of CpGs and genomic regions targeted by the MCCSeq capture panel design (.xIsx Excel spreadsheet format), and (2) a list of the targeted regions (.bed text file). (ZIP $4405 \mathrm{~kb})$

\section{Author details}

'Department of Human Genetics, McGill University, Montreal, Quebec, Canada. ${ }^{2}$ McGill University and Genome Quebec Innovation Centre, Montreal, Quebec, Canada. ${ }^{3}$ Team of Environmental Epidemiology Applied to Reproduction and Respiratory Health, Inserm U1209, CNRS, University Grenoble Alpes, Institute for Advanced Biosciences, Grenoble, France. ${ }^{4}$ Department of Human Genetics, The Wellcome Trust Sanger Institute, Wellcome Trust Genome Campus, Hinxton, Cambridge CB10 1HH, UK. ${ }^{5}$ Department of Haematology, University of Cambridge, Cambridge Biomedical Campus, Long Road, Cambridge CB2 OPT, UK. Institute of Nutrition and Functional Foods (INAF), Laval University, Québec, QC G1V 0A6, Canada. ${ }^{7}$ Genetic Variation and Human Diseases Unit, UMR-946, INSERM, Université Paris Diderot, Université Sorbonne Paris Cité, Paris, France.

${ }^{8}$ European Molecular Biology Laboratory, European Bioinformatics Institute,
Wellcome Genome Campus, Hinxton, Cambridge CB10 1SD, UK. ${ }^{9}$ UCL Cancer Institute, University College London, 72 Huntley Street, London WC1E 6BT, UK. ${ }^{10}$ Cardiovascular Epidemiology Unit, Department of Public Health and Primary Care, University of Cambridge, Strangeways Research Laboratory, Worts Causeway, Cambridge CB1 8RN, UK. ${ }^{11}$ Québec Heart and Lung Institute, Laval University, Québec, QC G1V 4G5, Canada. ${ }^{12}$ Pédiatrie, Centre Hospitalier Universitaire (CHU) Grenoble Alpes, Grenoble, France. ${ }^{13}$ National Health Service (NHS) Blood and Transplant, Cambridge Biomedical Campus, Long Road, Cambridge CB2 OPT, UK. ${ }^{14}$ Faculty of Science, Department of Molecular Biology, Radboud University, Nijmegen 6525GA, The Netherlands.

${ }^{15}$ British Heart Foundation Centre of Excellence, Division of Cardiovascular Medicine, Addenbrooke's Hospital, Hills Road, Cambridge CB2 0QQ, UK. ${ }^{16}$ The National Institute for Health Research Blood and Transplant Unit (NIHR BTRU) in Donor Health and Genomics, University of Cambridge, Strangeways Research Laboratory, Wort's Causeway, Cambridge CB1 8RN, UK.

Received: 25 April 2019 Accepted: 25 April 2019

Published online: 07 May 2019

\section{Reference}

1. Cheung, et al. Functional variation in allelic methylomes underscores a strong genetic contribution and reveals novel epigenetic alterations in the human epigenome. Genome Biol. 2017;18:50. https://doi.org/10.1186/ s13059-017-1173-7.

\footnotetext{
* Correspondence: tomi.pastinen@gmail.com; tomi.pastinen@mcgill.ca; elin.grundberg@mcgill.ca

'Warren A. Cheung, Xiaojian Shao and Andréanne Morin contributed equally to this work.

'Department of Human Genetics, McGill University, Montreal, Quebec,

Canada

Full list of author information is available at the end of the article
}

(c) The Author(s). 2019 Open Access This article is distributed under the terms of the Creative Commons Attribution 4.0 International License (http://creativecommons.org/licenses/by/4.0/), which permits unrestricted use, distribution, and reproduction in any medium, provided you give appropriate credit to the original author(s) and the source, provide a link to the Creative Commons license, and indicate if changes were made. The Creative Commons Public Domain Dedication waiver (http://creativecommons.org/publicdomain/zero/1.0/) applies to the data made available in this article, unless otherwise stated. 